PAPER • OPEN ACCESS

\section{External tuning of topological phase transitions induced by interaction driven mass renormalization}

To cite this article: Thies Jansen and Alexander Brinkman 2022 J. Phys.: Condens. Matter 34075601

View the article online for updates and enhancements.
You may also like

\begin{tabular}{l} 
- Fermi surfaces in Kondo insulators \\
Hsu Liu, Máté Hartstein, Gregory J \\
Wallace et al. \\
- $\frac{\text { Puzzle maker in } \mathrm{SmB}_{G} \text { : accompany-type }}{\text { valence fluctuation state }}$ \\
\hline Qi Wu and Liling Sun \\
- Intermediate-valence state of the Sm and \\
$\frac{\text { Eu in SmB }}{6}$ and EuCu Si $_{2}:$ neutron \\
$\frac{\text { Spectroscopy data and analysis }}{\text { P S Savchenkov, P A Alekseev, A }}$ \\
Podlesnyak et al.
\end{tabular}




\title{
External tuning of topological phase transitions induced by interaction driven mass renormalization
}

\author{
Thies Jansen* ${ }^{\circledR}$ and Alexander Brinkman \\ MESA+ Institute for Nanotechnology, University of Twente, The Netherlands \\ E-mail: t.jansen@utwente.nl
}

Received 1 September 2021, revised 31 October 2021

Accepted for publication 9 November 2021

Published 23 November 2021

\begin{abstract}
Electron-electron interactions can be useful for realizing new nontrivial topological phases of matter. Here, we show by means of a tight-binding model and mean field theory how electron-electron interactions can lead to a topological phase transition. By externally adding or removing electrons from the system a band inversion between two bands with different parity is induced. This leads to a topological nontrivial phase if spin-orbit coupling is present. Besides the toy-model illustrating this mechanism, we also propose $\mathrm{SmB}_{6}$ as a possible playground for experimentally realizing a topological phase transition by external tuning.
\end{abstract}

Keywords: electron-electron interactions, topology, tight-binding model, mean field theory, external tuning, spin-orbit coupling, $\mathrm{SmB}_{6}$

(Some figures may appear in colour only in the online journal)

\section{Introduction}

Electron-electron interactions in material systems give rise to rich phase diagrams and offer the possibility to tune between those phases by for example doping [1-3]. These phases are characterized by their transport properties, crystal structure and magnetic properties. Since the discovery of topological order of electronic band structures this also became an important phase of matter $[4,5]$. A topological nontrivial phase gives rise to dissipationless edge states and spin-momentum locking, which are interesting properties for new types of electronics. So far, topological insulators have been realized experimentally in $\mathrm{HgTe}$ quantum wells, bismuth selenide, bismuth telluride, antimony telluride and other related compounds [6-10]. All these materials are understood in the single

* Author to whom any correspondence should be addressed. of the Creative Commons Attribution 4.0 licence. Any further distribution of this work must maintain attribution to the author(s) and the title of the work, journal citation and DOI. particle picture where a band inversion leads to a non zero $\mathbb{Z}_{2}$ invariant [11].

Furthermore, since the discovery of topological quantum chemistry [12], most topological insulators based on the noninteracting picture are predicted and understood. So, in the search for new topological phases in materials, studying electron-electron interactions seems a promising direction.

Although some theoretical research has been done on topological phases in correlated materials [13-18] and topological phases induced by correlations [14, 19], an interaction driven topological phase is experimentally limited to the topological Kondo insulator $\mathrm{SmB}_{6}[20,21]$. On the theory side literature distinguishes two types of interaction driven topological phase transition: systems with and without spin-orbit coupling.

For example, Raghu et al [22], reported a system in which nearest and next-nearest neighbour Coulomb repulsion leads to spontaneous symmetry breaking, resulting in a system similar to Haldane [23]. In this system spin-orbit coupling is unnecessary for a topological nontrivial phase and can be replaced by electron-electron interactions. However, this particular system, and other similar systems only seem to be realized experimentally on optical lattices and not in materials [24-30]. Other theory studies also predict interaction induced 
topological phases, but spin-orbit coupling is still necessary to open up a gap [31-33]. Interactions are then important to renormalize different bands, resulting in a topological phase $[19,34]$.

Here we show how a system with spin-orbit interaction and a Hubbard $U$ like electron-electron interaction can lead to a topological phase transition. This simple toy-model shows the basic principle behind a 'interaction driven phase transition', which also applies to other systems were spin-orbit coupling is still a driving force. The model also shows how electron-electron interactions can be exploited to induce a topological phase transition by external tuning of the electron filling in a correlated band. We formulate requirements for such a system and discuss how $\mathrm{SmB}_{6}$ could be a possible playground for showing this mechanism experimentally.

\section{Tuning by electron filling}

In the presence of spin-orbit coupling, interactions can lead to a topological phase transition in the following way: the band populated with electrons will get an energy penalty, due to the repulsive electron-electron interactions, this pushes the band upwards. When the band is pushed through another band with a different parity and hybridizes, a topological phase transition occurs, provided that spin-orbit coupling is present. On the other hand, an attractive interaction would push the band downwards when adding electrons, which can also result in a topological phase transition. However, in this case the position of the Fermi level is unfavourable. So, here, the discussion is limited to a topological phase transition due to repulsive electron-electron interactions, which will be elaborated further on by means of the two-dimensional Bernevig-Hughes-Zhang (BHZ) model [35].

The BHZ model considers a two-dimensional square lattice, with the following four spin-orbit coupled orbitals: $|s, \uparrow\rangle$, $\left|p_{x}+i p_{y}, \uparrow\right\rangle,|s, \downarrow\rangle,\left|p_{x}-i p_{y}, \downarrow\right\rangle$. In this basis the BHZ Hamiltonian is obtained with the tight-binding method and takes the following form:

$$
\begin{aligned}
H_{\mathrm{BHZ}} & =\left(\begin{array}{cc}
h(k) & 0 \\
0 & h^{*}(-k)
\end{array}\right) \\
h(k) & =\left(\begin{array}{cc}
\epsilon_{s}-2 t_{s s}\left(\cos k_{x}+\cos k_{y}\right) & 2 t_{s p}\left(\sin k_{y}-i \sin k_{x}\right) \\
2 t_{s p}\left(\sin k_{y}+i \sin k_{x}\right) & \epsilon_{p}+2 t_{p p}\left(\cos k_{x}+\cos k_{y}\right)
\end{array}\right)
\end{aligned}
$$

where $\epsilon_{s}$ and $\epsilon_{p}$ are the onsite orbital energies of the $s$ and $p_{x} \pm i p_{y}$ orbital respectively. $t_{s s}, t_{s p}$ and $t_{p p}$ are the hopping integrals between the $s-s, s-p$ and $p-p$ orbital respectively. We set $t_{s s}=t_{p p}=1$ and $t_{s p}=0.5$. The values for the hopping parameters can be arbitrarily chosen and do not influence the qualitative findings of this work. We define $\epsilon_{p}=-m$ and $\epsilon_{s}=m$, such that the $p$ like band lies below the $s$ band. Consequently, the energy gap at the $\Gamma$ point is given by $\Delta=$ $2 m-4 t_{s s}-4 t_{p p}$. Band inversion between the $p$ and $s$ band takes place when this gap is smaller than zero. Similar reasoning for the case when the $p$ band lies above the $s$ band gives the requirement $\left|\frac{1}{2}\left(\epsilon_{s}-\epsilon_{p}\right)\right|<2 t_{s s}+2 t_{p p}$ for a topological non trivial phase $[11,35]$. So, the topological state of the system directly depends on the orbital energies of the $s$ and $p_{x} \pm i p_{y}$ orbitals. Interactions renormalize this term and force a topological phase transition.

Now we extend the BHZ model by including onsite electron-electron interactions in the lower lying $p$ like band:

$$
H_{U}=U \sum_{i} \hat{n}_{p, i, \uparrow} \hat{n}_{p, i \downarrow}
$$

where $\hat{n}_{\alpha, i, \sigma}=\hat{c}_{\alpha, i, \sigma}^{\dagger} \hat{c}_{\alpha, i, \sigma}$ is the density operator of orbital $\alpha$ at site $i$ with spin $\sigma$. In the mean field picture this term reduces to:

$$
H_{U}=U\left(\begin{array}{cccc}
0 & 0 & 0 & 0 \\
0 & \left\langle n_{p, \downarrow}\right\rangle & 0 & \chi \\
0 & 0 & 0 & 0 \\
0 & \chi^{\dagger} & 0 & \left\langle n_{p, \uparrow}\right\rangle
\end{array}\right),
$$

where $\left\langle n_{\alpha, \sigma}\right\rangle$ is the average occupation of orbital $\alpha$ with spin $\sigma$ and $\chi=\left\langle\hat{c}_{i \downarrow}^{\dagger} \hat{c}_{i \uparrow}\right\rangle$, stemming from the exchange term in the mean field approximation of the Hubbard Hamiltonian. The constant terms in this approximation are omitted. In the case of co-linear magnetic order $\chi=0$, on the other hand, for finite $\chi$ the spin up and spin down channels mix, resulting in a spin split band. The $\left\langle n_{\alpha, \sigma}\right\rangle$ terms renormalize the onsite orbital energy, as a consequence of electron-electron interactions.

This system is solved self-consistently under the constraint that:

$$
n=\sum_{\alpha, \sigma}\left\langle n_{\alpha, \sigma}\right\rangle
$$

where $n$ the average number of electrons per unit cell. Since one unit cell consist of two orbitals with double spin degeneracy, $n=1$ corresponds to quarter filling and $n=2$ to half filling. Here we use the electron filling as an extra 'knob' to tune the system through a topological phase transition, this is illustrated in figure 1 . For $n=1$ the system is in the trivial state with the Fermi level laying in the $p_{x} \pm i p_{y}$ band (blue). By adding extra electrons into the unit cell the electron onsite energy of the $p_{x} \pm i p_{y}$ band is renormalized and pushed through the $s$ band (red). As a result, the two bands invert. For $n=2$ the Fermi level shifts inside the gap and the system becomes a topological insulator.

For $n=1$ the spin degeneracy of the $p$ bands is lifted due the exchange term. This is reflected in a finite value of the order parameter $\chi$. For $n=2, \chi$ vanishes independently of the value of $U$ and $m$. This is shown in figure 2, which shows the value of $\chi$ for $m=5$ and different fillings $n$. In all the calculations $\chi=\chi^{\dagger}$ and the quantitative results are similar for different values of $m$. Figure 2 shows that only for large $U$ and half filling $\chi$ has a nonzero value.

For constructing an approximate phase diagram $\chi \approx 0$ is assumed. In this way, the phase boundaries for a band inversion are calculated using the requirement $0=2 m-4 t_{s s}-4 t_{p p}+$ $U\left\langle n_{p, \downarrow}\right\rangle$ as discussed above, but now with the renormalized mass term. This equation is solved self consistently for $m$ for a given $n$.

Figure 3 shows the phase diagram of the filling parameter $n$ versus the mass term $m$ for different values of $U$. When no electron-electron interactions are present, $U=0$, the phase 

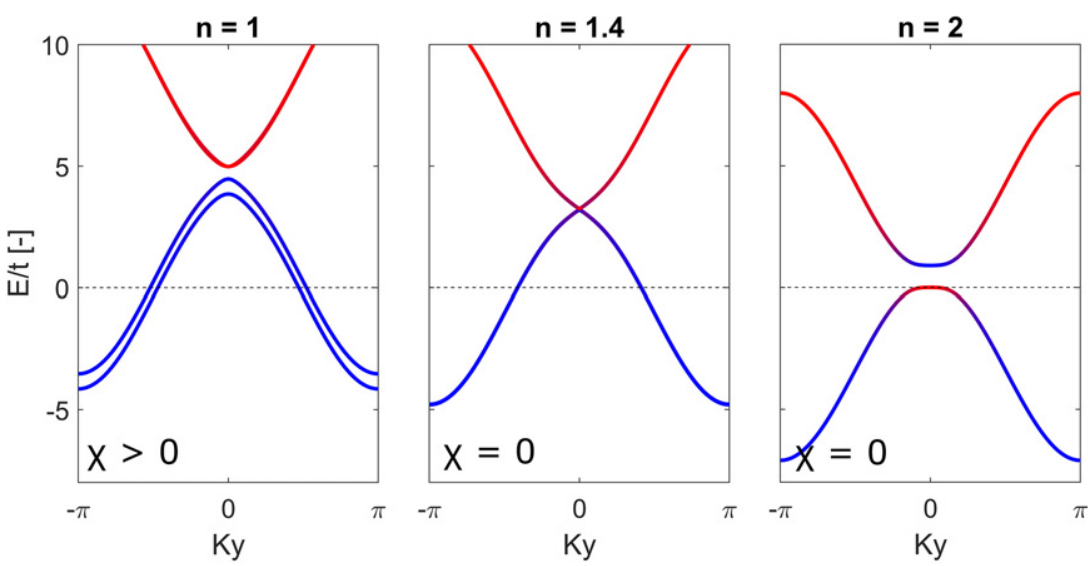

Figure 1. Band structure for $U=4, m=5.4$ and different fillings $n$. The Fermi energy is indicated by the dashed line. The blue color indicates a $p_{x} \pm i p_{y}$ like band, while a red color indicates an $s$ like band. By adding electrons the system can be tuned to a topological nontrivial phase. For $n=1$ the order parameter $\chi>0$, which results in a spin split band. For $n=1.4$ and $n=2 \chi$ vanishes.

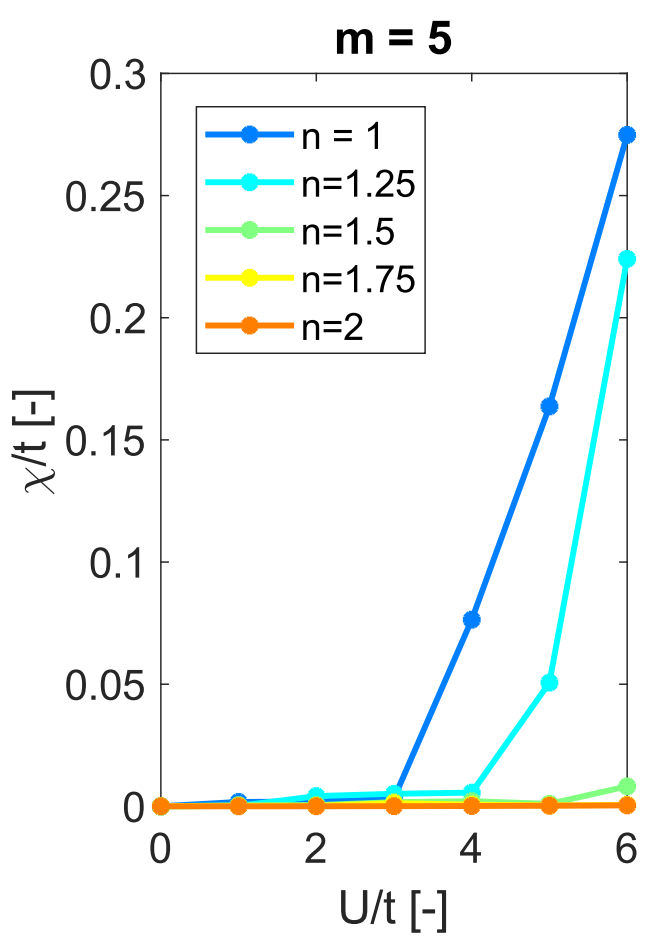

Figure 2. Order parameter $\chi$ as function for $U$ for different fillings $n$ at $m=5$.

diagram reflects the conventional requirement for a band inversion and, consequently, a topological phase at a filling of $n=2$. When interactions are turned on, the regime in which the system has a nontrivial topology is extended. Interactions also enables the possibility to tune the system through a topological phase transition by changing the filling at a fixed $m$. This is what is shown in figure 1 . Notice that only at an electron filling of $n=2$ the Fermi level lays inside the gap and the system can be considered a real topological insulator according to the Fu-Kane parity criterion. For other fillings the Fermi level lays inside a band and is said to be metallic. For half filling and large $U$, the phase diagram might be slightly inaccurate due to assumption of $\chi \approx 0$. A finite value of $\chi$ shift one spin

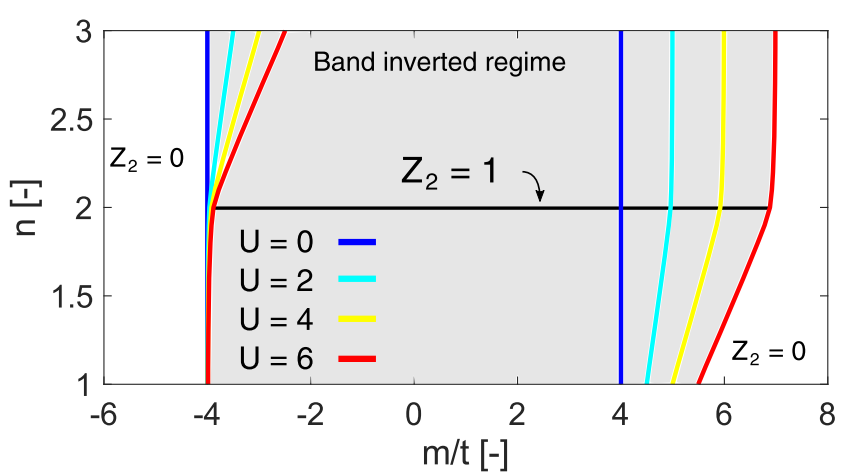

Figure 3. Phase diagram of $n$ versus $m$. The shaded region indicates the band inverted regime. When the bands are inverted and $n=2$ the system has a nontrivial topological phase. The phase boundary is indicated for different values of $U$.

band, which can results in a band inversion with a single spin band at slightly lower fillings. However, as shown in figure 2 the region of this inaccuracy is small.

\section{3. $\mathrm{SmB}_{6}$ as possible playground}

$\mathrm{SmB}_{6}$ is known as a topological Kondo insulator [20, 21]. The $d$ and $f$ bands cross and hybridize such that a gap opens. Since this systems involves correlated $d$ and $f$ bands, it might be an interesting system to study the mechanisms described above. To see the influence of the electron filling on this system the model by Alexanderov et al [36] is used and adapted. The model is similar, but the interactions are taken into account as described above. The Hubbard repulsion is set to $U_{d}=4 \mathrm{eV}$ and $U_{f}=6 \mathrm{eV}$ and the energy difference between the $d$ and $f$ bands is set to $12 \mathrm{eV}$. Figure 4 shows the electronic band structure for $n=4$ and $n=5$. For $n=4$ the system is a topological Kondo insulator, as indicated by the parity change at the high symmetry point $\mathrm{X}$. This would be the expected filling for $\mathrm{SmB}_{6}$. When the filling is changed the $d$ bands get filled and will be lifted out the $f$ bands, resulting in a transition to the trivial state. So, with doping and electrostatic gating $\mathrm{SmB}_{6}$ 
a.

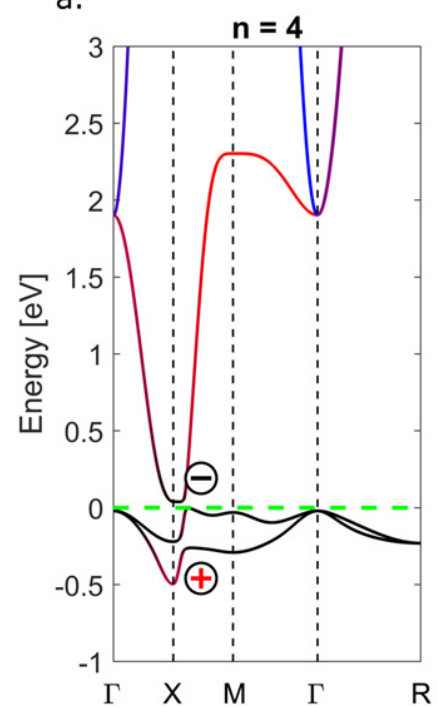

Figure 4. Band structure of $\mathrm{SmB}_{6}$ obtained with the adapted tight-binding model of reference [36]. For a filling of $n=4$ the material is a topological Kondo insulator (a). For $n=5$ the $d$ bands are lifted outside the $f$ bands and no band inversion takes place (b).

might be a good starting point in exploring the possibilities of tuning topological phase transitions due to electron-electron interactions.

\section{Discussion}

In view of the $\mathrm{BHZ}$ model, electron-electron interactions can alter the onsite energy of the different orbitals independently, such that effectively the $m$ parameter is tuned. Consequently, the system can be tuned externally through a topological phase transition by adding or removing electrons from the system by for example electrostatic gating or doping. The toy model presented here exploits this mechanism. However, the question remains if such a system is experimentally plausible. Figure 3 shows that the energy window of $m$ where topological phase transitions can occur is quite small, even for larger $U$. Furthermore, there is a large electron doping of one electron per unit cell needed in order to force the topological transition.

Based on these observations, an experimental realization of this toy-model should satisfy the following conditions: a large spin-orbit coupling, a large electron-electron interaction and two bands with different parity close in energy. With these requirements in mind, a system in which the Fermi level lays close to $d$ and $f$ bands seems a reasonable candidate.

However, there is one more important requirement: the bandwidth $(W)$ of the tuned band should be larger than the Hubbard repulsion $(U)$. If $U>W$ the spin degenerate band splits in two spin bands separated by an energy of $U$. In this case, adding electrons to the lower spin band will shift the position of the upper spin band, avoiding the topological gap opening around the Fermi level. In contrast, if $U<W$, the band stays spin degenerate and adding electrons coherently moves the band upward. Resulting in a topological gap opening around the Fermi level. This requirement excludes

materials like the perovskite materials $\mathrm{LaCuO}_{3}$ and $\mathrm{LaAgO}_{3}$. Electronic structure calculations without interactions of these materials show that the almost filled $e_{g}$ orbitals lay close to the La $4 f$ orbital band $[37,38]$. However, when interactions are taken into account, these systems become insulators and the $4 f$ electrons move way up in the conduction band [38]. This example shows that the transition metal oxides might not be the right material type, because the Hubbard $U$ is too large, resulting in spin split bands.

Another material class involving $d$ and $f$ bands are the Kondo insulators. Here we discussed the topological Kondo insulator $\mathrm{SmB}_{6}$ as a possible playground as a proof of principle system. Starting with the topological insulating phase in $\mathrm{SmB}_{6}$, adding electrons via doping could lift the $d$ bands out of the $f$ bands, electrostatic doping then might act as an extra knob to force a topological phase transition. This top-down approach has as an advantage that the bands are already close in energy and maybe a lot of doping can be avoided. However, it remains an open question if the energies $U_{d}, U_{f}$ and the energy difference between the $d$ and $f$ bands in $\mathrm{SmB}_{6}$ have to correct magnitude to externally tune a topological phase transition.

In summary, the presence of electron-electron interactions enables the electron filling to be used as an extra parameter to control a topological phase transition. More detailed calculations on different material systems should be done in order to see if this mechanism can be realized experimentally.

\section{Acknowledgments}

This work is part of the project TOPCORE (with Project Number OCENW.GROOT.2019.048) which is financed by the Dutch Research Council (NWO).

\section{Data availability statement}

The data that support the findings of this study are available upon reasonable request from the authors.

\section{ORCID iDs}

Thies Jansen (D) https://orcid.org/0000-0003-4545-2185

\section{References}

[1] Hemberger J, Krimmel A, Kurz T, Krug von Nidda H-A, Yu Ivanov V, Mukhin A A, Balbashov A M and Loidl A 2002 Structural, magnetic, and electrical properties of singlecrystalline $\mathrm{La}_{1-x} \mathrm{Sr}_{x} \mathrm{MnO}_{3}(0.4<x<0.85)$ Phys. Rev. B 66 094410

[2] Stanley H E, Kastner M A, Aharony A, Birgeneau R J and Coniglio A 1988 Magnetic phase diagram and magnetic pairing in doped $\mathrm{La}_{2} \mathrm{CuO}_{4}$ Phys. Rev. Lett. 60 1330-3

[3] Mott N F 1968 Metal-insulator transition Rev. Mod. Phys. 40 $677-83$

[4] Kane C L and Mele E J 2005 Quantum spin Hall effect in graphene Phys. Rev. Lett. 95226801 
[5] Kane C L and Mele E J $2005 Z_{2}$ topological order and the quantum spin Hall effect Phys. Rev. Lett. 95146802

[6] Hsieh D, Qian D, Wray L, Xia Y, Hor Y S, Cava R J and Hasan M Z 2008 A topological Dirac insulator in a quantum spin Hall phase Nature $\mathbf{4 5 2}$ 970-4

[7] Hsieh D et al 2009 Observation of time-reversal-protected single-Dirac-cone topological-insulator states in $\mathrm{Bi}_{2} \mathrm{Te}_{3}$ and $\mathrm{Sb}_{2} \mathrm{Te}_{3}$ Phys. Rev. Lett. 103146401

[8] Chen Y L et al 2009 Experimental realization of a threedimensional topological insulator, $\mathrm{Bi}_{2} \mathrm{Te}_{3}$ Science $\mathbf{3 2 5}$ $178-81$

[9] Xia Y et al 2009 Observation of a large-gap topologicalinsulator class with a single Dirac cone on the surface Nat. Phys. 5 398-402

[10] Ko"nig M, Wiedmann S, Brüne C, Roth A, Buhmann H, Molenkamp L W, Qi X-L and Zhang S-C 2010 Quantum spin Hall insulator state in HgTe quantum wells Science $\mathbf{3 1 8}$ 766-71

[11] Fu L and Kane C L 2007 Topological insulators with inversion symmetry Phys. Rev. B 76045302

[12] Barry B, Elcoro L, Cano J, Vergniory M G, Wang Z, Felser C, Aroyo M I and Andrei Bernevig B 2017 Topological quantum chemistry Nature $\mathbf{5 4 7} 298-305$

[13] Hohenadler M and Assaad F F 2014 Rashba coupling and magnetic order in correlated helical liquids Phys. Rev. B 90 245148

[14] Stephan R 2018 Interacting topological insulators: a review Rep. Prog. Phys. 81116501

[15] Rachel S and Le Hur K 2010 Topological insulators and Mott physics from the Hubbard interaction Phys. Rev. B 82 075106

[16] Budich J C, Trauzettel B and Sangiovanni G 2013 Fluctuationdriven topological Hund insulators Phys. Rev. B 87235104

[17] Xiao D, Zhu W, Ran Y, Nagaosa N and Okamoto S 2011 Interface engineering of quantum Hall effects in digital transition metal oxide heterostructures Nat. Commun. 2596

[18] Joseph M, Chua V and Gregory A 2014 Fiete topological order in a correlated three-dimensional topological insulator Phys. Rev. Lett. 112016404

[19] Zhang X, Zhang H, Wang J, Felser C and Zhang S-C 2012 Actinide topological insulator materials with strong interaction Science 335 1464-6

[20] Neupane M et al 2013 Surface electronic structure of the topological Kondo-insulator candidate correlated electron system $\mathrm{SmB}_{6}$ Nat. Commun. 42991

[21] Xu N et al 2013 Surface and bulk electronic structure of the strongly correlated system $\mathrm{SmB}_{6}$ and implications for a topological Kondo insulator Phys. Rev. B 88121102
[22] Raghu S, Qi X L, Honerkamp C and Zhang S C 2008 Topological Mott insulators Phys. Rev. Lett. 100156401

[23] Haldane F D M 1988 Model for a quantum Hall effect without landau levels: condensed-matter realization of the "parity anomaly' Phys. Rev. Lett. 61 2015-8

[24] Wu H-Q, Yuan-Yao H, Fang C, Meng Z Y and Lu Z-Y 2016 Diagnosis of interaction-driven topological phase via exact diagonalization Phys. Rev. Lett. 117066403

[25] Wen J, Andreas R, Joseph Wang C-C and Fiete G A 2010 Interaction-driven topological insulators on the Kagome and the decorated honeycomb lattices Phys. Rev. B 82075125

[26] Liu Q, Yao H and Ma T 2010 Spontaneous symmetry breaking in a two-dimensional Kagome lattice Phys. Rev. B 82045102

[27] Dauphin A, Müller M and Martin-Delgado M A 2016 Quantum simulation of a topological Mott insulator with Rydberg atoms in a Lieb lattice Phys. Rev. A 93043611

[28] Sun K, Yao H, Fradkin E and Steven A 2009 Kivelson topological insulators and nematic phases from spontaneous symmetry breaking in 2D Fermi systems with a quadratic band crossing Phys. Rev. Lett. 103046811

[29] Weeks C and Franz M 2010 Interaction-driven instabilities of a Dirac semimetal Phys. Rev. B 81085105

[30] Zhu W, Gong S-S, Zeng T-S, Fu L and Sheng D N 2016 Interaction-driven spontaneous quantum Hall effect on a Kagome lattice Phys. Rev. Lett. 117096402

[31] Budich J C, Thomale R, Li G, Laubach M and Zhang S-C 2012 Fluctuation-induced topological quantum phase transitions in quantum spin-Hall and anomalous-Hall insulators Phys. Rev. B 86201407

[32] Wang L, Dai X and Xie X C 2012 Interaction-induced topological phase transition in the Bernevig-Hughes-Zhang model Europhys. Lett. 9857001

[33] Wang L, Jiang H, Dai X and Xie X C 2012 Pole expansion of self-energy and interaction effect for topological insulators Phys. Rev. B 85235135

[34] Zhang P and Zhai H 2018 Topological Sachdev-Ye-Kitaev model Phys. Rev. B 97201112

[35] Bernevig B A, Hughes T L and Zhang S-C 2006 Quantum spin Hall effect and topological phase transition in $\mathrm{HgTe}$ quantum wells Science 314 1757-61

[36] Alexandrov V, Dzero M and Coleman P 2013 Cubic topological Kondo insulators Phys. Rev. Lett. 111226403

[37] Bacalis N C and Papaconstantopoulos D A 1988 Band structure and electron-phonon interaction of $\mathrm{LaAgO}_{3} J$. Supercond. 1 $175-80$

[38] Czyyk M T and Sawatzky G A 1994 Local-density functional and on-site correlations: the electronic structure of $\mathrm{La}_{2} \mathrm{CuO}_{4}$ and $\mathrm{LaCuO}_{3}$ Phys. Rev. B 49 14211-28 